\title{
Benzodiazepine use and risk of dementia: prospective population based study
}

\author{
@(®) OPEN ACCESS
}

\begin{abstract}
Sophie Billioti de Gage PhD student ${ }^{12}$, Bernard Bégaud professor ${ }^{123}$, Fabienne Bazin researcher ${ }^{12}$, Hélène Verdoux professor ${ }^{124}$, Jean-François Dartigues professor ${ }^{153}$, Karine Pérès researcher ${ }^{15}$, Tobias Kurth director of research ${ }^{167}$, Antoine Pariente associate professor $^{123}$
\end{abstract}

${ }^{1}$ Université Bordeaux Segalen, F-33000 Bordeaux, France; ${ }^{2}$ INSERM, U657, F-33000 Bordeaux; ${ }^{3} \mathrm{CHU}$ de Bordeaux, F-33000 Bordeaux; ${ }^{4} \mathrm{Centre}$ Hospitalier Charles Perrens, F-33000 Bordeaux; ${ }^{5}$ INSERM, U897, F-33000 Bordeaux; ${ }^{6}$ Division of Preventive Medicine, Brigham and Women's Hospital, Boston, MA, USA; ${ }^{7}$ INSERM, U708-Neuroepidemiology, F-33000 Bordeaux,

\begin{abstract}
Objective To evaluate the association between use of benzodiazepines and incident dementia.

Design Prospective, population based study.

Setting PAQUID study, France.

Participants 1063 men and women (mean age 78.2 years) who were free of dementia and did not start taking benzodiazepines until at least the third year of follow-up.

Main outcome measures Incident dementia, confirmed by a neurologist.

Results During a 15 year follow-up, 253 incident cases of dementia were confirmed. New use of benzodiazepines was associated with an increased risk of dementia (multivariable adjusted hazard ratio 1.60, $95 \%$ confidence interval 1.08 to 2.38 ). Sensitivity analysis considering the existence of depressive symptoms showed a similar association (hazard ratio $1.62,1.08$ to 2.43). A secondary analysis pooled cohorts of participants who started benzodiazepines during follow-up and evaluated the association with incident dementia. The pooled hazard ratio across the five cohorts of new benzodiazepine users was 1.46 (1.10 to 1.94). Results of a complementary nested case-control study showed that ever use of benzodiazepines was associated with an approximately $50 \%$ increase in the risk of dementia (adjusted odds ratio $1.55,1.24$ to 1.95) compared with never users. The results were similar in past users (odds ratio $1.56,1.23$ to 1.98 ) and recent users $(1.48,0.83$ to 2.63 ) but reached significance only for past users.
\end{abstract}

Conclusions In this prospective population based study, new use of benzodiazepines was associated with increased risk of dementia. The result was robust in pooled analyses across cohorts of new users of benzodiazepines throughout the study and in a complementary case-control study. Considering the extent to which benzodiazepines are prescribed and the number of potential adverse effects of this drug class in the general population, indiscriminate widespread use should be cautioned against.

\section{Introduction}

Primarily indicated for treating the symptoms of anxiety and sleep disorders over short periods, ${ }^{1}$ benzodiazepines are widely prescribed in developed countries. ${ }^{2}{ }^{3}$ In France, 30\% of people aged 65 years and over use benzodiazepines. ${ }^{4}$ They are used by more than $20 \%$ of people aged 65 and over in Canada and Spain and by around $15 \%$ of those in Australia. ${ }^{5-7}$ Benzodiazepine use is less widespread but still high in elderly people in the United States and the United Kingdom. ${ }^{8}{ }^{9}$ Consumption of benzodiazepines is often chronic, ${ }^{2}{ }^{10}$ and many people take them for years despite the existence of good practice guidelines suggesting that the duration should be limited to a few weeks. ${ }^{10-12}$

The short term effects of benzodiazepines on cognition are well known. ${ }^{13-16}$ They are mediated through an agonist action on receptors of $\gamma$ aminobutyric acid A, a major inhibitory neurotransmitter in the brain. However, the long term adverse effects of benzodiazepines on cognition are still debated.

Studies focusing on the association between benzodiazepine use and dementia or cognitive decline in elderly people have shown conflicting results. ${ }^{17}$ Some found an increased risk of dementia or cognitive impairment in benzodiazepine users, ${ }^{918-21}$ whereas others were not conclusive or reported a potential protective effect. ${ }^{22-27}$ In previous studies, the timing of exposure to benzodiazepines in relation to the outcome event allowed for the possibility of reverse causation. Insomnia, depression, and anxiety (the main indications for prescribing benzodiazepines) can be prodromal symptoms of dementia. ${ }^{28}$ 
Dementia is already responsible for a major societal burden worldwide. With more than 81 million cases expected in $2040,{ }^{29}$ this burden will become even greater in the coming decades. ${ }^{30}$ As treatment options remain limited, identifying factors contributing to dementia is critical. The objective of this study was to assess the association between starting benzodiazepines and risk of subsequent dementia in a well defined population based cohort of elderly people with available follow-up of up to 20 years.

\section{Methods}

\section{Participants, design, and settings}

We studied participants in the prospective PAQUID cohort study, which assesses normal and pathological brain ageing. Its methods and design have been described in detail elsewhere. ${ }^{31}$ Briefly, a representative sample of 3777 community dwelling people aged 65 years and over between 1987 and 1989 were randomly selected from the general population of the Gironde and Dordogne, two administrative areas in southwest France. ${ }^{31}$ Follow-up visits took place every two or three years, and information up to the 20 year visit is available. At baseline and at each follow-up visit, trained neuropsychologists collected detailed information on personal characteristics, sociodemographics, life habits, health conditions, drug use, functional abilities, depressive symptoms, and cognition during face to face interviews.

In the study reported here, we used a cohort and a nested case-control study design. In the cohort (main) analysis, we introduced an observation period of three to five years before the start of follow-up. People were eligible to participate if they were free from dementia at the five year follow-up visit $\left(\mathrm{T}_{5}\right)$ and reported use of benzodiazepines for the first time on that date (fig $1 \Downarrow$ ). Thus, participants did not use benzodiazepines at least up to the three year follow-up visit. This period was essential to allow adjustment for factors associated with starting benzodiazepines, including cognitive decline from inclusion in the PAQUID study $\left(\mathrm{T}_{0}\right)$ to the three year follow-up visit, allaying concerns about confounding by indication, and to limit and assess the possibility of a reverse causation that was suspected in previously published studies. In the nested case-control study, we included all eligible participants in the PAQUID study.

\section{Exposure definition and measurement}

Data on drug use, including benzodiazepine use, were collected with a standardised questionnaire at each follow-up visit. In addition, participants or their usual caregivers were asked about prescribed and non-prescribed drugs used regularly during the previous two weeks. The interviewer then validated drug use by visual inspection of the participant's medicine packs. The neuropsychologists who carried out the face to face interviews were unaware of the hypothesis of our study.

We classified all eligible participants as new benzodiazepine users or non-users according to ascertainment of exposure at $\mathrm{T}_{5}$ (fig $1 \Downarrow$ ). The exposure group comprised participants without declared benzodiazepine use at $\mathrm{T}_{0}$ and $\mathrm{T}_{3}$ and declaration of use at $\mathrm{T}_{5}$, so they constituted new users of benzodiazepines between $\mathrm{T}_{3}$ and $\mathrm{T}_{5}$. In the main analysis, we did not consider subsequent exposure to benzodiazepines when estimating the association between benzodiazepine use and occurrence of dementia. Participants without any declared use at $\mathrm{T}_{0}, \mathrm{~T}_{3}$, and $\mathrm{T}_{5}$ served as the reference group.

We considered all benzodiazepines and similar drugs available in France between 1988 and 2006 (alprazolam, bromazepam, chlordiazepoxide, clobazam, clonazepam, clorazepate, clotiazepam, diazepam, estazolam, flunitrazepam, loflazepate, loprazolam, lormetazepam, nitrazepam, nordazepam, prazepam, oxazepam, temazepam, tetrazepam, tofizopam, triazolam, zolpidem, and zopiclone).

\section{Outcome definition and measurement}

At each follow-up, trained psychologists systematically assessed dementia on the basis of the Diagnostic and Statistical Manual of Mental Disorders, third edition, revised (DSM-III-R). Neurologists further examined suspected cases to confirm the diagnosis; our main outcome was neurologist confirmed dementia. As the follow-up visits were two or three years apart, we assigned the midpoint of the time interval between two follow-up visits as the index date for dementia. We determined incident cases of dementia by using information from the eight year follow-up visit $\left(\mathrm{T}_{8}\right)$, indicating any new occurrence of dementia after baseline $\left(\mathrm{T}_{5}\right)$.

\section{Covariates}

In addition to age and sex, which are risk factors both for benzodiazepine use and for dementia, the covariates used for adjustment at the baseline for this study $\left(\mathrm{T}_{5}\right)$ included factors considered to be associated with both benzodiazepine use and risk of dementia ${ }^{32}$ :33: educational level (schooling duration $\geq 7$ years $v<7$ years), marital status (single or not), wine consumption (regular consumption $v$ no consumption), existence of diabetes mellitus or high blood pressure (defined according to patients' use of antidiabetic agents or antihypertensive drugs at study baseline $\left(\mathrm{T}_{5}\right)$ ), use of statins, use of platelet inhibitors or oral anticoagulants, and cognitive decline. For cognitive decline, three different tests were considered: the mini-mental state examination, ${ }^{34}$ the Benton visual retention test,${ }^{35}$ and the Isaacs set test. ${ }^{36}$ For these three tests, we considered the difference between the scores obtained at $\mathrm{T}_{3}$ and at $\mathrm{T}_{0}$ (inclusion in the PAQUID study) as a quantitative variable. Depressive symptoms were assessed by using the Center for Epidemiologic Studies depression scale, ${ }^{37}$ categorised according to the threshold validated for the French population with a cut-off score of $\geq 17$ for men and $\geq 23$ for women, ${ }^{38}$ defining the presence of significant depressive symptoms.

\section{Statistical analysis Cohort, main analysis}

We compared the characteristics of new users of benzodiazepines with those of non-users by using numbers and percentages for qualitative variables and median and interquartile range for quantitative variables. Figure $1 \Downarrow$ details the follow-up scheme and design.

We used Kaplan-Meier curves and log-rank tests for univariable dementia-free survival analyses in exposed and non-exposed groups. We used multivariable adjusted Cox proportional hazards models to evaluate the association between new use (starting between $\mathrm{T}_{3}$ and $\mathrm{T}_{5}$ ) of benzodiazepines and risk of incident dementia, adjusting for potential confounding factors. The dependent variable was the occurrence of incident dementia. We adjusted the models for the following potential confounding factors (covariates): age, sex, education level, marital status, regular wine consumption, change in the mini-mental state examination between $\mathrm{T}_{0}$ and $\mathrm{T}_{3}$, and use of antidiabetic agents, antihypertensive drugs, statins, and platelet inhibitors or oral anticoagulants. As depression is thought to be a potential prodromal symptom of dementia, ${ }^{39}{ }^{40}$ we did not consider depressive symptoms as a potential confounder in our main 
analysis. However, we included information on the presence of depressive symptoms in a sensitivity analysis because depression has also been described as a risk factor for dementia. ${ }^{41} \mathrm{We}$ calculated person time from the date of the PAQUID five year follow-up visit (baseline of our study) to the estimated date of dementia, death, or loss to follow-up or the date of the 20 year follow-up visit, whichever came first. We verified the proportionality assumption of the Cox model by evaluating Cox-Snell residuals and found no violation.

We evaluated whether the association between benzodiazepine use and dementia was modified by age (80-84 or $\geq 85 v 70-79$ years), sex, or schooling duration ( $\geq 7 v<7$ years). To test for statistical significant effect modification, we used the likelihood ratio test contrasting the main model with a model including appropriate interaction terms.

\section{Cohort, secondary analysis}

To account for potential time varying effects on the association between benzodiazepine use and risk of dementia, we considered new cohorts of benzodiazepines users between the baseline of our study (PAQUID $\mathrm{T}_{5}$ ) and the following 15 years of follow-up (PAQUID $\mathrm{T}_{8}$ to $\mathrm{T}_{20}$ ) in a second analysis. We chose this approach as we believe that a Cox model updating information on exposure would not allow for a correct causal inference structure. ${ }^{42}$ At "baseline" and each of the four follow-up time points $\left(\mathrm{T}_{8}, \mathrm{~T}_{10}, \mathrm{~T}_{13}\right.$, and $\left.\mathrm{T}_{15}\right)$, we created cohorts of new users (participants reporting benzodiazepine use for the first time at the specific follow-up visit) and followed each cohort until a censoring event occurred or until the end of the study, whichever came first. We then evaluated the association of each of these cohorts with the risk of subsequent dementia compared with participants who did not start benzodiazepines at a given time point, adjusting for the above mentioned confounders including information on depressive symptoms. The information on confounders was updated to the respective follow-up time point. Finally, we pooled the five cohorts by using a fixed effect model.

\section{Nested case-control analysis}

Participants still followed at $\mathrm{T}_{8}$, without dementia before this date and with an accurate date of diagnosis of dementia, were eligible for a nested case-control analysis in the PAQUID cohort. We defined cases as participants with an incident diagnosis of dementia from $\mathrm{T}_{8}$ onwards, as in the cohort study. We considered all participants without a diagnosis of dementia at the time point when a case was diagnosed (index date) as controls. Among these, we randomly selected up to four controls and matched them with each case by age ( \pm 2 years) and sex. Participants could be used as controls for cases and later selected as cases if they presented with an incident diagnosis of dementia (incidence density sampling).

For the nested case-control study, we did not exclude any prevalent benzodiazepine exposure. We first classified participants as ever users (if at least one use of benzodiazepine was declared before the index date) and never users (no declaration of benzodiazepine use before index date). Among ever users, we then identified recent users (reported use at the follow-up visit preceding the index date but never before) and past users (reported use at least three visits before the index date or earlier).

We used conditional logistic regression to evaluate the association between benzodiazepine use and risk of dementia. We built two series of models, each considering one of the definitions retained for exposure to benzodiazepines. We did analyses using similar adjustment to that used for the main cohort analysis, including depressive symptoms. Models not including information on depressive symptoms showed similar associations. Potential confounding variables were measured at three follow-up time points (seven or eight years) before the index date.

We used the SAS statistical package (SAS 9.3 for Windows) for all analyses. All reported $P$ values are two tailed, and we considered $\mathrm{P}<0.05$ to be statistically significant.

\section{Results \\ Cohort analysis Population}

Of the 3777 participants in the PAQUID study, 2084 were still in active follow-up at the five year visit $\left(\mathrm{T}_{5}\right.$, baseline for this study). We excluded 154 participants with prevalent dementia at the five year follow-up visit, 735 participants with a history of benzodiazepine use or prevalent use, and 132 with missing or questionable information on history of benzodiazepine use. The sample thus included 1063 participants free of dementia at $\mathrm{T}_{5}$ and without prevalent use of benzodiazepines up until $\mathrm{T}_{3}$ (fig $2 \Downarrow)$. Among the 1063 eligible participants, 95 (8.9\%) reported consumption of a benzodiazepine at study baseline $\left(\mathrm{T}_{5}\right)$, indicating new use between $\mathrm{T}_{3}$ and $\mathrm{T}_{5}$. Table $1 \Downarrow$ shows characteristics of participants included in the analysis according to benzodiazepine exposure status. Compared with non-users $(n=968)$, new users of benzodiazepines $(n=95)$ were more likely to have shorter school duration $(66 \% v 77 \%$ with duration $\geq 7$ years), to be single or widowed (52\% v 41\%), to have more significant depressive symptoms (16\% v 4\%), to use antihypertensive drugs (74\% v 58\%), and to use platelet inhibitors or oral anticoagulants $(15 \% v 6 \%)$ and consumed wine less regularly $(63 \% v 73 \%)$. No difference existed between new users of benzodiazepines and non-users in terms of age, sex, diabetes mellitus, statin use, and cognitive evolution between inclusion in the PAQUID study $\left(\mathrm{T}_{0}\right)$ and the three year follow-up visit $\left(\mathrm{T}_{3}\right)$.

\section{Main analysis}

During the 15 year follow-up (median 6.2 (interquartile range 2.6-12.3) years), 253 (23.8\%) cases of dementia were confirmed-30 (32\%) in benzodiazepine users and $223(23.0 \%)$ in non-users. Compared with non-use, new use of benzodiazepines between $\mathrm{T}_{3}$ and $\mathrm{T}_{5}$ was associated with a shorter dementia-free survival in the study population (crude survival analysis, log-rank test $\mathrm{P}=0.03$ ) (fig $3 \Downarrow$ ). The incidence rate of dementia during the 15 year follow-up was 4.8 per 100 person years in the exposed group compared with 3.2 per 100 person years in the non-exposed group. Compared with participants who did not report use of benzodiazepines at baseline $(n=968)$, starting benzodiazepines between $\mathrm{T}_{3}$ and $\mathrm{T}_{5}(\mathrm{n}=95)$ was associated with a significantly increased risk of dementia (hazard ratio $1.60,95 \%$ confidence interval 1.08 to 2.38 ), after adjustment for potential confounding factors. When further adjusted for depressive symptoms, this result was unchanged (hazard ratio 1.62, 1.08 to 2.43) (table $2 \Downarrow$ ). Use of the Isaacs or the Benton test instead of the mini-mental state examination yielded similar results (hazard ratios 1.67 and 1.68).

Out of the 1063 participants included, 57 (5\%) had missing values for at least one of the 10 variables used for adjustment in the main analysis-8 (8\%) in the exposed group and $49(5 \%)$ in the non-exposed group $(\mathrm{P}=0.17)$. We did not find any significant effect modification of the association between starting benzodiazepines and incident dementia by age ( $\mathrm{P}$ for 
interaction $=0.10)$, sex $(\mathrm{P}$ for interaction $=0.23)$, or duration of schooling $(\mathrm{P}$ for interaction $=0.43$ ).

\section{Secondary analysis}

In the secondary analysis, we created benzodiazepine new user and non-user cohorts at baseline $\left(\mathrm{T}_{5}\right)$ and the follow-up time points $\mathrm{T}_{8}, \mathrm{~T}_{10}, \mathrm{~T}_{13}$, and $\mathrm{T}_{15}$. This added a total of 116 new users during follow-up to the 95 new users at baseline. We then pooled the association between new use of benzodiazepine and risk of incident dementia across the five cohorts (baseline and four follow-up cohorts) by using a fixed effect model. The pooled multivariable adjusted hazard ratio of dementia was 1.40 (1.06 to 1.85). Additional control for symptoms of depression did not alter this result (hazard ratio $1.46,1.10$ to 1.94 ) (fig $4 \Downarrow$ ).

\section{Nested case-control analysis}

One thousand six hundred and thirty-three participants still followed at $\mathrm{T}_{8}$ were eligible for the nested case-control analysis (fig $5 \Downarrow$ ). We identified 467 cases of dementia and 1810 controls for the nested case-control analysis (which did not exclude prevalent users at $\mathrm{T}_{0}$ or $\mathrm{T}_{3}$, unlike in the cohort analysis). Table $3 \Downarrow$ shows characteristics of cases and controls. Ever users had an increased risk of dementia (adjusted odds ratio 1.55, 1.24 to 1.95). We found similar associations in past users (adjusted odds ratio $1.56,1.23$ to 1.98 ) and recent users (adjusted odds ratio $1.48,0.83$ to 2.63 ), but the result was significant only for past users (table $4 \Downarrow$ ).

\section{Discussion}

In this large, prospective, population based study of elderly people who were free of dementia and did not use benzodiazepines until at least the third year of follow-up, new use of benzodiazepines was associated with a significant, approximately $50 \%$ increase in the risk of dementia. This result remained stable after adjustment for potential confounding factors, including cognitive decline before starting benzodiazepine and clinically significant symptoms of depression. It also remained robust when we pooled five cohorts of new benzodiazepine users throughout the 15 year follow-up period and in a complementary nested case-control study.

\section{Comparison with other studies}

Our findings are consistent with three previous case-control studies that also showed an increased risk of dementia in benzodiazepine users. Two of them were done in Taiwan using health insurance data of people aged 45 years and older and found an increased risk of dementia in chronic users ( $>6$ months) (adjusted odds ratio 1.24, 1.01 to 1.53$)^{18}$ and current users (adjusted odds ratio 2.71, 2.46 to 2.99). ${ }^{19}$ A nested case-control study among French people aged 65 and over showed an increased risk of dementia in former users (adjusted odds ratio 2.3, 1.2 to 4.5). ${ }^{21}$ However, the maximum length of follow-up in those studies was eight years, ${ }^{18}{ }^{19} 21$ raising concerns that prodomal symptoms of dementia could have influenced benzodiazepine use. ${ }^{28}$ The results of the Caerphilly prospective cohort showed an increase in the risk of dementia in people who ever used benzodiazepines (adjusted odds ratio 2.94, 1.16 to 7.46) up to 22 years of follow-up. The results of that study also suggested that the potential excess risk of dementia associated with benzodiazepine use may apply to a population with low benzodiazepine consumption. However, benzodiazepine users could not be classified as new starters and the sample size was too small to allow for meaningful subgroup analyses (for example, 12 participants had exposure of four years or more among the 93 with dementia). ${ }^{9}$

In contrast to our results, two studies did not find an increased risk of dementia among elderly people using benzodiazepines. The results of one (case-control) study, however, showed a similar effect estimate to ours, but this did not reach statistical significance (adjusted odds ratio 1.5, 0.6 to 3.4), probably owing to the small sample size. ${ }^{22}$ The other (cohort) study, suggested a beneficial effect of benzodiazepine use on dementia (unadjusted odds ratio 0.34) but considered past users as members of the reference group, which could have biased the results. ${ }^{27} \mathrm{~A}$ more detailed comparison of studies that have explored the potential association between benzodiazepine use and the risk of dementia is summarised in the supplementary (web extra) table.

Our study included a markedly larger number of participants than did the previous long term study on the topic, ${ }^{9}$ and we incorporated a run-in time of at least three years to allow adjustment for factors associated with starting benzodiazepines, thereby reducing the possibility of reverse causation. In that sense, an excess risk observed during the early phase of exposure could be congruent with a reverse causation bias, which would not be the case if the increase in risk appeared long after the start of exposure.

Benzodiazepines could also be seen as an early risk marker for dementia that might highlight a particular at risk background in patients, but without playing any causal role in the occurrence of the disease. For example, persistent anxiety in middle age has been shown to be associated with a greater risk of dementia in elderly people. ${ }^{43}$ Hence, benzodiazepine use may be a marker of this scenario and might help to identify people at increased risk of, and not already on the causal pathway leading to, dementia. However, in our study, two observations argue against this hypothesis: the strength of association did not increase across the five cohorts of new users (which would be expected in the event of reverse causation, as the time from benzodiazepine use to development of dementia is shorter), and the association between new use of benzodiazepines and dementia increased after about seven years of follow-up (fig $3 \Downarrow$ ). However, reverse causation cannot be entirely ruled out as an alternative explanation of our findings.

\section{Strengths and limitations}

This study has several strengths. Firstly, it was based on a long follow-up period allowing assessment of the delayed effects of exposure to benzodiazepine, which was not possible in previous studies (except one ${ }^{9}$ ). Secondly, we defined incident dementia by applying validated criteria (DSM-III-R) ascertained by senior neurologists who were blind to the study hypothesis. Thirdly, trained interviewers prospectively recorded and validated data on drug exposure by visual inspection of the patients' medicine. Fourthly, restricting analyses to new users of benzodiazepine after at least a three to five year "run-in" period allowed us to adjust for factors strongly associated with starting benzodiazepines. Lastly, the study was carried out in a large representative cohort of elderly participants, ${ }^{31}$ with adjustment on a large number of potential confounders of the benzodiazepine-dementia association. ${ }^{2} 33233$

The study also has several limitations. Firstly, we had a limited number of new users at baseline $(n=95)$, thereby limiting the power for subgroup analyses and ruling out the possibility of assessing individual benzodiazepines. However, the literature contains no suggestion that specific benzodiazepines have differential effects on cognition or dementia. ${ }^{23}$ Secondly, we 
could not adjust separately for anxiety and sleep disorders, both putative dementia prodromes, owing to the lack of specific measurement of these symptoms in the PAQUID programme. However, the Center for Epidemiologic Studies depression scale includes these items in computation of its global score, and entering three separate scores in the model could have raised a concern about colinearity, as depressive symptoms are often associated with sleep disorders and anxiety, especially in elderly people. Lastly, people who had missing information for benzodiazepine use during the three to five year run-in period of this study were not included, as they could not be ascertained for exposure status. As these missing data were linked to unperformed follow-up, which has been related to subsequent higher risk of dementia, ${ }^{44}$ a potential selection bias could have occurred. However, these ineligible participants had a lower schooling level and were more likely to live alone, two factors known to increase the risk of both benzodiazepine use and incident dementia. Therefore, a potential selection bias due to exclusion of people with missing information for exposure before the index date would have tended to decrease the strength of the association. The disparity in the incidence of dementia over a comparable time window was similar to that seen in the participants included.

\section{Implication for clinical practice and public health}

Benzodiazepines remain useful for the treatment of acute anxiety states and transient insomnia. ${ }^{45}{ }^{46}$ However, increasing evidence shows that their use may induce adverse outcomes, mainly in elderly people, such as serious falls and fall related fractures. ${ }^{47} 48$ Our data add to the accumulating evidence that use of benzodiazepines is associated with increased risk of dementia, which, given the high and often chronic consumption of these drugs in many countries, ${ }^{10} 4950$ would constitute a substantial public health concern. Therefore, physicians should carefully assess the expected benefits of the use of benzodiazepines in the light of these adverse effects and, whenever possible, limit prescription to a few weeks as recommended by the good practice guidelines. ${ }^{1}$ In particular, uncontrolled chronic use of benzodiazepines in elderly people should be cautioned against.

\section{Unanswered questions and future research}

Further research should explore whether long term use of benzodiazepines in people under 65 is also associated with an increased risk of dementia and should study possible correlations between dosage or cumulative length of exposure and dementia.

\section{Conclusion}

The findings of this large prospective population based study show that new use of benzodiazepines is associated with an approximately $50 \%$ increase in the risk of dementia. The results remained robust after control for potential confounders, in pooled analysis across the follow-up time, and in a nested case-control study. Considering the extent to which benzodiazepines are now prescribed, physicians and regulatory agencies should consider the increasing evidence of the potential adverse effects of this drug class for the general population.

We thank Catherine Helmer (INSERM U897 and Université Bordeaux Segalen, Bordeaux, France) for her valuable comments on the results and manuscript and Marie Tournier (INSERM U657 and Université Bordeaux Segalen, Bordeaux, France) for her comments on the protocol and analytic approach.
Contributors: SBdG did the statistical analysis, interpreted the results, and drafted the manuscript. BB contributed to the design of the study, interpreted the results, and participated in the writing and editing of the manuscript. FB oversaw and contributed to the statistical analysis. HV contributed to definition of benzodiazepine exposure and depressive symptoms and critically revised the manuscript. J-FD and KP provided all necessary information about the PAQUID cohort, prodromal dementia, and diagnosis of dementia and revised the manuscript. TK and AP equally contributed to the study design and protocol development, interpretation of results, and manuscript writing and editing. All the authors made a significant contribution to the research and the development of the manuscript and approved the final version. SBdG and $\mathrm{BB}$ are the guarantors.

Funding: This research was conducted by the INSERM U657 research team co-funded by INSERM (Institut National de la Santé et de la Recherche Médicale) and Université Bordeaux Segalen. Additional support was provided by a 2010 grant from IRESP (Institut de Recherche en Santé Publique) acting on behalf of the French Ministry of Health (Direction Générale de la Santé, Direction de la Recherche, des Études, de l'Évaluation et des Statistiques); by a 2011 grant from the French Ministry of Health (Direction Générale de la Santé); and by Caisse Nationale des Travailleurs Salariés, Régime Social des Indépendants, Caisse Nationale de Solidarité pour l'Autonomie, and Institut National de Prévention et d'Education pour la Santé.

Competing interests: All authors have completed the Unified Competing Interest form at http://www.icmje.org/coi_disclosure.pdf (available on request from the corresponding author) and declare: that this study has been funded only by academic research funds; no financial relationships with any organisation or company that might have an interest in the submitted work in the previous three years; no other relationships or activities that could appear to have influenced the submitted work. SBdG is a part time researcher in the INSERM 657 Unit, and her salary is paid by IRESP. BB has received investigator initiated research funding from the French Health Ministry (2011); he is chair of the scientific committee for two pharmacoepidemiological studies conducted by the contract research organisation LA-SER (London): one on medicines used in osteoarthritis, the other on the use of homeopathic remedies by French practitioners. HV has received funding from the French Association de Recherche sur le Cancer (ARC) for a research project on psychotropic drugs and breast cancer. J-FD has received grants from IPSEN and Novartis and honorariums from Merck, Serono, Novartis, and Ipsen. TK has received investigator initiated research funding from the French National Research Agency, the US National Institutes of Health, the Migraine Research Foundation, and the Parkinson's Disease Foundation; he has received honorariums from Allergan, the American Academy of Neurology, and Merck for educational lectures and from MAP

Pharmaceutical for contributing to a scientific advisory panel; he receives honorariums from the $B M J$ for editorial services. AP has received investigator initiated research funding from the Agence Française de Sécurité Sanitaire des Produits de Santé (AFSSAPS) and from IRESP; he has participated in research funded by the Fonds de la Recherche en Santé du Québec (FRSQ), the Réseau Québécois de Recherche sur l'Utilisation des Médicaments (RQRUM), the European Union (FP7 grants), the Innovative Medicines Initiative (IMI grant), and Sanofi-Aventis.

Ethical approval: The PAQUID study was approved by the ethics committee of the University of Bordeaux Segalen (France) in 1988, and each participant provided written informed consent.

Data sharing: No additional data available.

This work is licensed under the Creative Commons Attribution-NonCommercial 3.0 Unported License. To view a copy of this license, visit http://creativecommons.org/licenses/by-nc/3.0/ or write to Creative Commons, 444 Castro Street, Suite 900, Mountain View, CA, 94041, USA. 


\section{What is already known on this topic}

Prescription of benzodiazepines in elderly people is widespread and often chronic in many developed countries, regardless of good practice guidelines

Benzodiazepines can have delayed adverse effects on cognition (cognitive decline and dementia), as reported in several case-control studies and a few cohort studies

\section{What this study adds}

Benzodiazepine exposure was associated with dementia, even after a long follow-up period

Adjustment for factors strongly associated with starting benzodiazepines or considered to be markers for a prodrome of early dementia did not alter the association

1 European Medicines Agency. Summary of product characteristics for benzodiazepines as anxiolytics or hypnotics. 1994. www.ema.europa.eu/docs/en_GB/document_library/ Scientific_guideline/2009/09/WC500003774.pdf.

2 Sonnenberg CM, Bierman EJ, Deeg DJ, Comijs HC, van Tilburg W, Beekman AT. Ten-year trends in benzodiazepine use in the Dutch population. Soc Psychiatry Psychiatr Epidemiol 2012;47:293-301.

3 Lagnaoui R, Depont F, Fourrier A, Abouelfath A, Begaud B, Verdoux H, et al. Patterns and correlates of benzodiazepine use in the French general population. Eur $\mathrm{J}$ Clin Pharmacol 2004;60:523-9.

4 Fourrier A, Letenneur L, Dartigues JF, Moore N, Begaud B. Benzodiazepine use in an elderly community-dwelling population: characteristics of users and factors associated with subsequent use. Eur J Clin Pharmacol 2001;57:419-25.

5 Hogan DB, Maxwell CJ, Fung TS, Ebly EM. Prevalence and potential consequences of benzodiazepine use in senior citizens: results from the Canadian Study of Health and Aging. Can J Clin Pharmacol 2003;10:72-7.

6 Carrasco-Garrido P, Jimenez-Garcia R, Astasio-Arbiza P, Ortega-Molina P, de Migue AG. Psychotropics use in the Spanish elderly: predictors and evolution between years 1993 and 2003. Pharmacoepidemiol Drug Saf 2007;16:449-57.

7 Windle A, Elliot E, Duszynski K, Moore V. Benzodiazepine prescribing in elderly Australian general practice patients. Aust N Z J Public Health 2007;31:379-81.

8 Paulose-Ram R, Safran MA, Jonas BS, Gu Q, Orwig D. Trends in psychotropic medication use among U.S. adults. Pharmacoepidemiol Drug Saf 2007;16:560-70.

9 Gallacher J, Elwood P, Pickering J, Bayer A, Fish M, Ben-Shlomo Y. Benzodiazepine use and risk of dementia: evidence from the Caerphilly Prospective Study (CaPS). $J$ Epidemiology Community Health 2012;66:869-73.

10 Egan M, Moride Y, Wolfson C, Monette J. Long-term continuous use of benzodiazepines by older adults in Quebec: prevalence, incidence and risk factors. J Am Geriatr Soc 2000;48:811-6.

11 Neutel $\mathrm{Cl}$. The epidemiology of long-term benzodiazepine use. Int Rev Psychiatry 2005:17:189-97.

12 Vinkers $\mathrm{CH}$, Olivier $\mathrm{B}$. Mechanisms underlying tolerance after long-term benzodiazepine use: a future for subtype-selective GABA(A) receptor modulators? Adv Pharmacol SCi 2012;2012:416864.

13 Lister RG. The amnesic action of benzodiazepines in man. Neurosci Biobehav Rev 1985:9:87-94

14 Ghoneim MM, Mewaldt SP. Benzodiazepines and human memory: a review. Anesthesiology 1990;72:926-38.

15 Curran HV. Tranquillising memories: a review of the effects of benzodiazepines on human memory. Biol Psychol 1986;23:179-213.

16 Buffett-Jerrott SE, Stewart SH. Cognitive and sedative effects of benzodiazepine use. Curr Pharm Des 2002;8:45-58.

17 Verdoux $\mathrm{H}$, Lagnaoui $\mathrm{R}$, Begaud $\mathrm{B}$. Is benzodiazepine use a risk factor for cognitive decline and dementia? A literature review of epidemiological studies. Psychol Med 2005;35:307-15.

18 Wu CS, Wang SC, Chang IS, Lin KM. The association between dementia and long-term use of benzodiazepine in the elderly: nested case-control study using claims data. $A m J$ Geriatr Psychiatry 2009;17:614-20.

19 Wu CS, Ting TT, Wang SC, Chang IS, Lin KM. Effect of benzodiazepine discontinuation on dementia risk. Am J Geriatr Psychiatry 2011;19:151-9.

20 Paterniti S, Dufouil C, Alperovitch A. Long-term benzodiazepine use and cognitive decline in the elderly: the Epidemiology of Vascular Aging Study. J Clin Psychopharmacol 2002;22:285-93.

21 Lagnaoui R, Begaud B, Moore N, Chaslerie A, Fourrier A, Letenneur L, et al. Benzodiazepine use and risk of dementia: a nested case-control study. J Clin Epidemio 2002;55:314-8

22 Lagnaoui R, Tournier M, Moride Y, Wolfson C, Ducruet T, Begaud B, et al. The risk of cognitive impairment in older community-dwelling women after benzodiazepine use. Age Ageing 2009;38:226-8

23 Hanlon JT, Horner RD, Schmader KE, Fillenbaum GG, Lewis IK, Wall WE Jr, et al. Benzodiazepine use and cognitive function among community-dwelling elderly. Clin Pharmacol Ther 1998;64:684-92.

24 Dealberto MJ, McAvay GJ, Seeman T, Berkman L. Psychotropic drug use and cognitive decline among older men and women. Int $J$ Geriatr Psychiatry 1997;12:567-74.

25 Boeuf-Cazou O, Bongue B, Ansiau D, Marquie JC, Lapeyre-Mestre M. Impact of long-term benzodiazepine use on cognitive functioning in young adults: the VISAT cohort. Eur $J$ Clin Pharmacol 2011;67:1045-52.

26 Allard J, Artero S, Ritchie K. Consumption of psychotropic medication in the elderly: a re-evaluation of its effect on cognitive performance. Int J Geriatr Psychiatry 2003;18:874-8.

27 Fastbom J, Forsell Y, Winblad B. Benzodiazepines may have protective effects against Alzheimer disease. Alzheimer Dis Assoc Disord 1998;12:14-7.
28 Amieva H, Le Goff M, Millet X, Orgogozo JM, Peres K, Barberger-Gateau P, et al. Prodromal Alzheimer's disease: successive emergence of the clinical symptoms. Ann Neurol 2008;64:492-8.

29 Ferri CP, Prince M, Brayne C, Brodaty H, Fratiglioni L, Ganguli M, et al. Global prevalence of dementia: a Delphi consensus study. Lancet 2005;366:2112-7.

30 Sousa RM, Ferri CP, Acosta D, Albanese E, Guerra M, Huang Y, et al. Contribution of chronic diseases to disability in elderly people in countries with low and middle incomes: a 10/66 Dementia Research Group population-based survey. Lancet 2009;374:1821-30.

31 Dartigues JF, Gagnon M, Barberger-Gateau P, Letenneur L, Commenges D, Sauvel C, et al. The Paquid epidemiological program on brain ageing. Neuroepidemiology 1992;11(suppl 1):14-8

$32 \mathrm{Li} \mathrm{J}$, Wang YJ, Zhang M, Xu ZQ, Gao CY, Fang CQ, et al. Vascular risk factors promote conversion from mild cognitive impairment to Alzheimer disease. Neurology 2011;76:1485-91.

33 Ballard C, Gauthier S, Corbett A, Brayne C, Aarsland D, Jones E. Alzheimer's disease. Lancet 2011;377:1019-31.

34 Folstein MF, Folstein SE, McHugh PR. "Mini-mental state": a practical method for grading the cognitive state of patients for the clinician. J Psychiatr Res 1975;12:189-98.

35 Benton AL. Abbreviated versions of the visual retention test. J Psychol 1972;80:189-92.

36 Isaacs B, Kennie AT. The set test as an aid to the detection of dementia in old people. Br J Psychiatry 1973;123:467-70.

37 Radloff $L$. The CES-D scale: a self-report depression scale for research in the general population. Appl Psychol Meas 1977:1:385-401.

38 Fuhrer R, Rouillon F. La version française de l'échelle CES-D (Center for epidemiologic studies-depression scale): description et traduction de l'échelle d'autoévaluation. Psychiatrie \& Psychobiologie 1989;4:163-6.

39 Muliyala KP, Varghese M. The complex relationship between depression and dementia. Ann Indian Acad Neurol 2010;13(suppl 2):S69-73.

40 Li G, Wang LY, Shofer JB, Thompson ML, Peskind ER, McCormick W, et al. Temporal relationship between depression and dementia: findings from a large community-based 15-year follow-up study. Arch Gen Psychiatry 2011;68:970-7.

41 Geerlings MI, den Heijer T, Koudstaal PJ, Hofman A, Breteler MM. History of depression, depressive symptoms, and medial temporal lobe atrophy and the risk of Alzheimer disease. Neurology 2008;70:1258-64.

42 Hernan MA, Brumback B, Robins JM. Marginal structural models to estimate the causa effect of zidovudine on the survival of HIV-positive men. Epidemiology 2000;11:561-70.

43 Johansson L, Guo X, Waern M, Ostling S, Gustafson D, Bengtsson C, et al. Midlife psychological stress and risk of dementia: a 35 -year longitudinal population study. Brain 2010;133:2217-24.

44 Jacqmin-Gadda H, Fabrigoule C, Commenges D, Dartigues JF. A 5-year longitudinal study of the mini-mental state examination in normal aging. Am J Epidemiol 1997; 145:498-506.

45 Glass J, Lanctot KL, Herrmann N, Sproule BA, Busto UE. Sedative hypnotics in older people with insomnia: meta-analysis of risks and benefits. BMJ 2005;331:1169.

46 Dell'osso B, Lader M. Do benzodiazepines still deserve a major role in the treatment of psychiatric disorders? A critical reappraisal. Eur Psychiatry 2012; published online 19 April.

47 Pariente A, Dartigues JF, Benichou J, Letenneur L, Moore N, Fourrier-Reglat A. Benzodiazepines and injurious falls in community dwelling elders. Drugs Aging 2008;25:61-70.

48 Pierfitte C, Macouillard G, Thicoipe M, Chaslerie A, Pehourcq F, Aissou M, et al. Benzodiazepines and hip fractures in elderly people: case-control study. $B M J$ 2001;322:704-8.

49 Jorm AF, Grayson D, Creasey H, Waite L, Broe GA. Long-term benzodiazepine use by elderly people living in the community. Aust N Z J Public Health 2000;24:7-10.

50 Briot M. Le bon usage des médicaments psychotropes. Office Parlementaire d'Evaluation des Politiques de Santé, 2006

\section{Accepted: 04 September 2012}

\section{Cite this as: BMJ 2012:345:e6231}

This is an open-access article distributed under the terms of the Creative Commons Attribution Non-commercial License, which permits use, distribution, and reproduction in any medium, provided the original work is properly cited, the use is non commercial and is otherwise in compliance with the license. See: http://creativecommons.org/licenses/bync/2.0/ and http://creativecommons.org/licenses/by-nc/2.0/legalcode. 


\section{Tables}

Table 1/ Baseline characteristics of participants from PAQUID study included in cohort analysis, according to benzodiazepine use. Values are numbers (percentages) unless stated otherwise

\begin{tabular}{lcc} 
Characteristics & Benzodiazepine new users ( $\mathbf{n}=95)$ & Benzodiazepine non-users ( $\mathbf{n}=968)$ \\
Median (interquartile range) follow-up (years) & $6.1(2.2-10.4)$ & $6.2(2.6-12.5)$ \\
\hline Dementia* & $30(32)$ & $223(23.0)$ \\
\hline Female sex & $54(57)$ & $474(49.0)$ \\
\hline Age (years): & $35(37)$ & $385(39.8)$ \\
\hline $70-79$ & $26(27)$ & $240(24.8)$ \\
\hline $80-84$ & $34(36)$ & $343(35.4)$ \\
\hline$\geq 85$ & $63(66)$ & $745(77.0)$ \\
\hline Schooling duration $\geq 7$ years & $49(52)$ & $394(40.7)$ \\
\hline Single or widowed & $57 / 90(63)$ & $680 / 932(73.0)$ \\
\hline Wine consumption & $15 / 93(16)$ & $38 / 944(4.0)$ \\
\hline Significant depressive symptoms $\dagger^{*}$ & $70(74)$ & $562(58.1)$ \\
\hline High blood pressureł & $7(7)$ & $31(8.4)$ \\
\hline Diabetes mellitus $\S$ & $5(5)$ & $56(3.7)$ \\
\hline Statin use & $14(15)$ & \\
\hline Platelet inhibitor or oral anticoagulant use & & $0(-1-1)$ \\
\hline Median (interquartile range) cognitive evolution trend: & $0(-1-1)$ & $0(-3-3)$ \\
\hline MMSE score difference between $\mathrm{T}_{3}$ and $\mathrm{T}_{0}$ & $0(-2-3)$ & $0(-1-2)$ \\
\hline Isaacs score difference between $\mathrm{T}_{3}$ and $\mathrm{T}_{0}$ & $0(-2-1)$ & \\
\hline Benton score difference between $\mathrm{T}_{3}$ and $\mathrm{T}_{0}$ & &
\end{tabular}

MMSE=mini-mental state examination.

*According to Diagnostic and Statistical Manual of Mental Disorders, third edition, revised criteria.

†Based on Center for Epidemiologic Studies depression scale (score $\geq 17$ for men; $\geq 23$ for women).

$\ddagger$ According to use of antihypertensive drugs.

§According to use of antidiabetic agents. 
Table 2| Association between new use of benzodiazepine with incident dementia in PAQUID study. Values are numbers (percentages) unless stated otherwise

\begin{tabular}{|c|c|c|c|}
\hline & Incident dementia & No dementia during follow-up & Hazard ratio $(95 \% \mathrm{Cl})$ \\
\hline Analysis adjusted for age ${ }^{\star}$ & $(n=253)$ & $(n=810)$ & \\
\hline Benzodiazepine non-users & $223(88)$ & $745(92.0)$ & 1.00 \\
\hline Benzodiazepine new users & $30(12)$ & $65(8.0)$ & 1.59 (1.09 to 2.34$)$ \\
\hline Main analysis $†$ & $(n=240)$ & $(n=766)$ & \\
\hline Benzodiazepine non-users & $211(88)$ & $708(92.4)$ & 1.00 \\
\hline Benzodiazepine new users & $29(12)$ & $58(7.6)$ & $1.60(1.08$ to 2.38$)$ \\
\hline Complementary analysis $† \ddagger$ & $(n=231)$ & $(n=752)$ & \\
\hline Benzodiazepine non-users & $203(88)$ & $695(92.4)$ & 1.00 \\
\hline Benzodiazepine new users & $28(12)$ & $57(7.6)$ & $1.62(1.08$ to 2.43$)$ \\
\hline
\end{tabular}

\section{${ }^{*}$ At baseline $\left(T_{5}\right)$}

†Adjusted for age, sex, schooling duration, singleness, wine consumption, use of antihypertensive drugs, use of antidiabetic agents, use of statins, use of platelet inhibitors or oral anticoagulants, and mini-mental state examination evolution between inclusion $\left(\mathrm{T}_{0}\right)$ and three year follow-up visit $\left(\mathrm{T}_{3}\right)$.

$\ddagger$ Adjusted for significant depressive symptoms according Center for Epidemiologic Studies depression scale (score $\geq 17$ for men; $\geq 23$ for women) at baseline ( $T_{5}$ ). 
Table 3| Characteristics of cases and controls included in nested case-control study from PAQUID study. Values are numbers (percentages)

\begin{tabular}{lcc} 
Characteristics & Cases $(\mathbf{n}=\mathbf{4 6 7})$ & Controls $(\mathbf{n}=\mathbf{1 8 1 0})$ \\
Benzodiazepine ever users & $233(50)$ & $741(40.9)$ \\
\hline Benzodiazepine recent users & $17(4)$ & $52(2.9)$ \\
\hline Benzodiazepine past users & $216(46)$ & $689(38.1)$ \\
\hline Schooling duration $\geq 7$ years & $298(64)$ & $1339(74.0)$ \\
\hline Single or widowed & $240 / 456(53)$ & $1012 / 1784(56.7)$ \\
\hline Wine consumption & $267 / 454(59)$ & $1066 / 1777(60.0)$ \\
\hline Significant depressive symptoms* & $46 / 450(10)$ & $153 / 1778(8.6)$ \\
\hline High blood pressure† & $274 / 456(60)$ & $1125 / 1784(63.1)$ \\
\hline Diabetes mellitusł & $29 / 456(6)$ & $96 / 1784(5.4)$ \\
\hline Statin use & $26 / 456(6)$ & $73 / 1784(4.1)$ \\
\hline Platelet inhibitor or oral anticoagulant use & $32 / 456(7)$ & $164 / 1784(9.2)$
\end{tabular}

*Based on Center for Epidemiologic Studies depression scale (score $\geq 17$ for men; $\geq 23$ for women). †According to use of antihypertensive drugs.

$\ddagger$ According to use of antidiabetic agents. 
Table 4| Risk of dementia associated with benzodiazepine use in nested case control study of 1633 elderly people form PAQUID study. Values are numbers (percentages) unless stated otherwise

\begin{tabular}{|c|c|c|c|}
\hline & Cases & Controls & Odds ratio $(95 \% \mathrm{Cl})$ by drug use \\
\hline \multicolumn{4}{|l|}{ Ever use analysis } \\
\hline Univariate analysis*: & $(n=467)$ & $(n=1810)$ & \\
\hline Benzodiazepine non-users & $175(37)$ & $845(46.7)$ & 1.00 \\
\hline Benzodiazepine ever users & $233(50)$ & $741(40.9)$ & 1.54 (1.24 to 1.93$)$ \\
\hline Missing exposure & $59(13)$ & $224(12.4)$ & $1.30(0.93$ to 1.81$)$ \\
\hline Multivariable analysis ${ }^{*} \dagger:$ & $(n=449)$ & $(\mathrm{n}=1771)$ & \\
\hline Benzodiazepine non-users & $174(39)$ & $844(47.7)$ & 1.00 \\
\hline Benzodiazepine ever users & $233(52)$ & $738(41.7)$ & 1.55 (1.24 to 1.95$)$ \\
\hline Missing exposure & $42(9)$ & $189(10.7)$ & $1.04(0.71$ to 1.53$)$ \\
\hline \multicolumn{4}{|l|}{ Recent/past use analysis } \\
\hline Univariate analysis*: & $(n=467)$ & $(n=1810)$ & \\
\hline Benzodiazepine non-users & $175(37)$ & $845(46.7)$ & 1.00 \\
\hline Benzodiazepine recent users & $17(4)$ & $52(2.9)$ & $1.58(0.90$ to 2.78$)$ \\
\hline Benzodiazepine past users & $216(46)$ & $689(38.1)$ & $1.54(1.23$ to 1.93$)$ \\
\hline Missing exposure & $59(13)$ & $224(12.4)$ & $1.30(0.93$ to 1.81$)$ \\
\hline Multivariable analysis ${ }^{*}+:$ & $(n=449)$ & $(\mathrm{n}=1771)$ & \\
\hline Benzodiazepine non-users & $174(39)$ & $844(47.7)$ & 1.00 \\
\hline Benzodiazepine recent users & $17(4)$ & $52(2.9)$ & $1.48(0.83$ to 2.63$)$ \\
\hline Benzodiazepine past users & $216(48)$ & $686(38.7)$ & 1.56 (1.23 to 1.98$)$ \\
\hline Missing exposure & $42(9)$ & $189(10.7)$ & $1.04(0.71$ to 1.53$)$ \\
\hline
\end{tabular}

*Matched for age and sex.

†Adjusted for schooling duration, singleness, wine consumption, use of antihypertensive drugs, use of antidiabetic agents, use of statins, use of platelet inhibitors or oral anticoagulants, and significant depressive symptoms according to Center for Epidemiologic Studies depression scale (score $\geq 17$ for men; $\geq 23$ for women), 7 or 8 years before index date. 


\section{Figures}

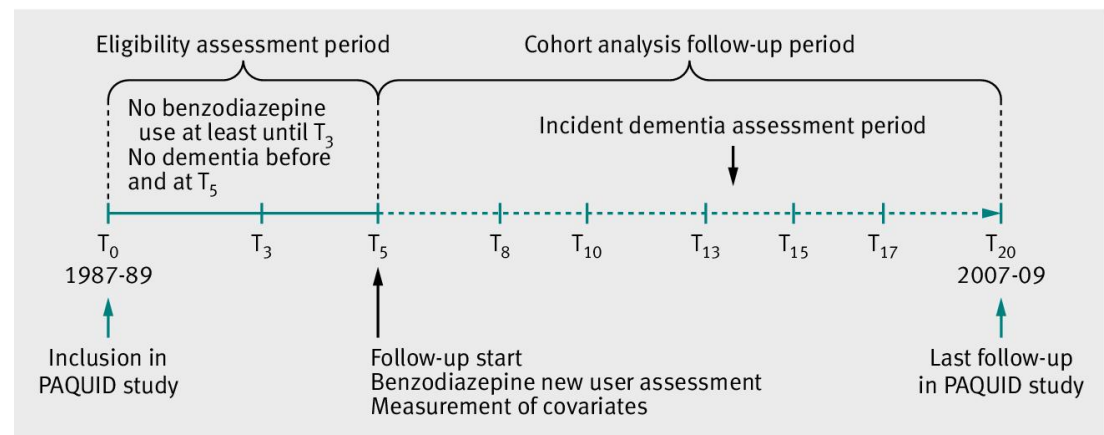

Fig 1 Follow-up scheme and study design for primary cohort analysis

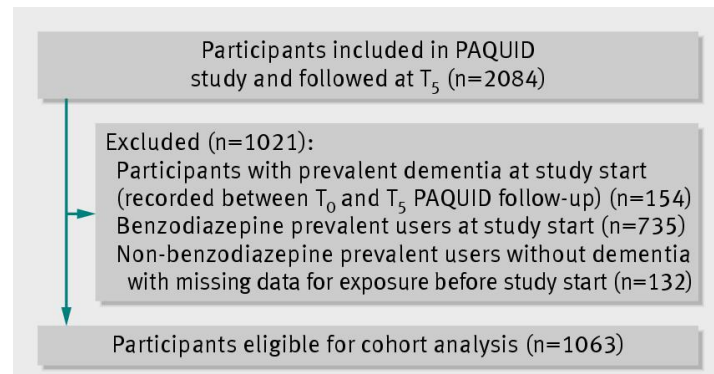

Fig 2 Identification of participants from PAQUID study followed up at $T_{5}$ (follow-up at year 5 of PAQUID study) and eligible for main cohort analysis (no dementia and no prevalent benzodiazepine use)

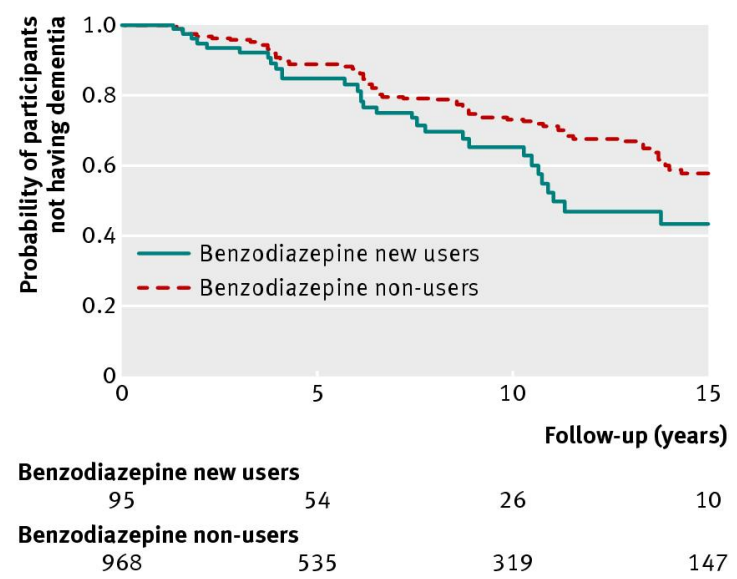

Fig 3 Dementia-free survival in PAQUID study, in new benzodiazepine users and non-users at baseline $\left(T_{5}\right)$ 


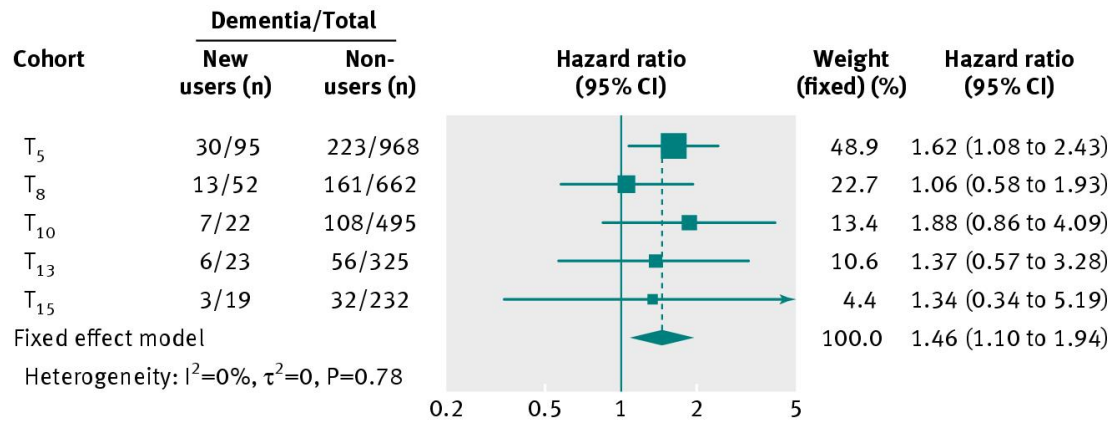

Fig 4 Pooled associations between new benzodiazepine use at follow-up years 5 (cohort $T_{5}$ ), 8 (cohort $T_{8}$ ), 10 (cohort $T_{10}$ ), 13 (cohort $T_{13}$ ), and 15 (cohort $T_{15}$ ) and risk of subsequent dementia. Results adjusted for age, sex, schooling duration, singleness, wine consumption, use of antihypertensive drugs, use of antidiabetic agents, use of statins, use of platelet inhibitors or oral anticoagulants, depressive symptoms, and mini-mental state examination evolution between inclusion ( $\left.T_{0}\right)$ and 3 year follow-up visit $\left(\mathrm{T}_{3}\right)$

Participants included in PAQUID study $(n=3777)$

Excluded $(n=2144)$ :

Participants lost from follow-up or died before $T_{8}$

$(n=1899)$

Had dementia before $T_{8}(n=70)$

Date of dementia unknown $(n=175)$

Subjects eligible for nested case-control analysis $(n=1633)$

Fig 5 Identification of participants from PAQUID study eligible for nested case-control analysis 